\title{
Trends in the Cost-effectiveness of Chemoprevention for Breast Cancer-2001 to 2015
}

\author{
Austin M Coe, BA ${ }^{1}$ and Katherine D Crew, MD, MS ${ }^{2-4}$
}

\begin{abstract}
1. Masters Student, Department of Epidemiology, Mailman School of Public Health, Columbia University, New York, NY, US; 2. Assistant Professor of Medicine and Epidemiology, Department of Epidemiology, Mailman School of Public Health, Columbia University, New York, NY, US; 3. Department of Medicine, Division of Hematology/ Oncology, College of Physicians and Surgeons, Columbia University, New York, NY, US; 4. Herbert Irving Comprehensive Cancer Center, Columbia University, New York, NY, US
\end{abstract}

DOI: http://doi.org/10.17925/OHR.2015.11.02.104

\begin{abstract}
Breast cancer is the most commonly diagnosed cancer among US women and costs of treatment are estimated to be over $\$ 16.5$ billion annually. The use of chemopreventive agents for the primary prevention of breast cancer has been an available option for women at high risk for breast cancer since 1998, however, uptake has been low. Cost-effectiveness analyses are used to determine whether a given intervention will be beneficial for a specific population while maintaining costs below a standard threshold for health systems. A number of cost-effectiveness analyses on the use of the chemopreventive drugs, tamoxifen and raloxifene, among women at high risk for breast cancer have been published over the past 15 years. These studies have used diverse methodologies to determine which, if any, high-risk women would benefit from the use of chemoprevention. The results of these cost-effectiveness analyses have been highly varied, with about half finding that chemoprevention is cost-effective for women who meet high-risk criteria for breast cancer. The sparse literature available in this field highlights the need for a more comprehensive analysis of the cost-effectiveness of chemoprevention that takes into account the most recently available information on costs and outcomes.
\end{abstract}

\section{Keywords}

Breast cancer, chemoprevention, cost-effectiveness, tamoxifen, raloxifene, anastrozole, exemestane

Disclosures: Austin M Coe, BA, and Katherine D Crew, MD, MS, have nothing to disclose in relation to this article. No funding was received in the publication of this article. open Access: This article is published under the Creative Commons Attribution Noncommercial License, which permits any noncommercial use, distribution, adaptation, and reproduction provided the original author(s) and source are given appropriate credit.

Received: August 28, 2015 Accepted: October 26, 2015 Citation: Oncology \& Hematology Review, 2015;11(2):104-9

Correspondence: Katherine D Crew, MD, MS, Assistant Professor of Medicine and Epidemiology, Herbert Irving Comprehensive Cancer Center, Columbia University,

161 Fort Washington Ave, 10-1072, New York, NY 10032, US. E: kd59@cumc.columbia.edu

Breast cancer is the most commonly diagnosed cancer and is the second most common cause of cancer-related death among women in the US, leading to over 40,000 deaths annually. 1,2 The cost of diagnosing, treating, and providing long-term follow-up care for these patients has a major impact on health systems. Estimates of the average annual costs to a woman diagnosed with breast cancer range from $\$ 12,828$ to $\$ 27,693$, and a study by Mariotto et al. estimated total costs to be over $\$ 16.5$ billion in 2010.3,4 Annual costs of breast cancer care are projected to surpass $\$ 20$ billion by $2020 .{ }^{4}$ These costs are likely to go up as screening rates increase and detection methods improve. Healthcare providers, faced with higher rates of potential false positive results and overdiagnosis with screening mammography, must make critical decisions about how to manage patients that are identified as high risk for breast cancer. ${ }^{5}$ Given national concerns about the increasing costs of breast cancer diagnoses and treatment, chemoprevention is a potentially attractive option for preventing breast cancer before it actually manifests.
Use of chemopreventive agents for the primary prevention of breast cancer has been an option for high-risk women in the US since 1998, when the Food and Drug Administration (FDA) first approved the selective estrogen receptor modulator (SERM), tamoxifen. ${ }^{6}$ Since then another SERM, raloxifene, has been approved by the FDA for the prevention of breast cancer and two aromatase inhibitors (Als), exemestane and anastrozole, have been shown to reduce breast cancer risk among high-risk postmenopausal women., ${ }^{6,7}$ While these drugs work differently and have a diverse range of side effects, they all have the potential to significantly reduce the risk for breast cancer for high-risk women; with a relative risk (RR) of 0.71 for tamoxifen, 0.35 for exemestane, and 0.47 for anastrozole versus placebo. ${ }^{8-10}$ Updated results from the Study of Tamoxifen and Raloxifene (STAR) trial showed a RR of 1.19 for raloxifene compared with tamoxifen, or $81 \%$ of the efficacy. ${ }^{11}$ Despite the potential of these pills to greatly reduce breast cancer-related costs, their uptake among high-risk women, defined as having a 5-year invasive breast cancer risk for at least $1.67 \%$ based upon the Gail Model, has been low. ${ }^{12}$ 
Eligibility for chemoprevention in the clinical trials included a $1.67 \%$ 5-year risk of breast cancer or a $20 \%$ lifetime risk.9,13,14 It is estimated that 10 million, or $15.5 \%$, of US women aged 35-79 meet this high-risk threshold, however, fewer than $5 \%$ of women who are offered chemoprevention agree to take it. ${ }^{12,15}$ Much has been written on this low uptake, however, it is primarily attributed to the risk for side effects and lack of knowledge about chemoprevention options among primary care providers. ${ }^{12,16,17}$

A number of cost-effectiveness analyses have been published over the past decade with more than half indicating that chemoprevention is a cost-effective option for the prevention of breast cancer among certain groups of high-risk women. These studies simulated a population of women with varied breast cancer risk levels and demographic backgrounds and compared the cost of chemoprevention to the "effectiveness" of the intervention, usually measured as quality-adjusted life years (QALYS). In the analyses evaluated, chemoprevention would be considered cost-effective if the ratio of cost to QALYS saved was below a threshold of \$50,000-100,000 and cost-saving if treatment costs were less than placebo or no treatment. The use of chemoprevention drugs was considered "dominated", or always less cost-effective than other options, if treatment was more expensive with less favorable outcomes than an alternative treatment or placebo, or if use of chemoprevention had a negative impact on patient survival. QALYs were generally calculated by multiplying the life years saved by a utility score, representing a subject's preference for a particular outcome, and aggregating these values over the lifetime. Unless otherwise specified, all analyses reviewed in this article used the Gail model to estimate risk for breast cancer in the model population, because this model was used to determine eligibility for the majority of chemoprevention trials conducted in the US. However, the Gail model is not well-validated in certain minority populations (e.g. Hispanics) and may underestimate breast cancer risk in women with a strong family history of the disease. For the purpose of comparability, we have noted when cost-effectiveness was analyzed for women at a $1.67 \% 5$-year risk for breast cancer, as this is the definition for high risk used in the chemoprevention trials conducted in the US. ${ }^{17}$ While long-term followup trials have not shown an overall survival benefit to chemoprevention, many cost-effectiveness analyses indicate that the treatment may still be cost-effective or cost-saving. Several of these studies have also indicated that the benefits of using chemoprevention outweigh the risks for side effects in these groups. ${ }^{18-21}$

Articles for this review were identified by performing searches through PubMed using several terms, including "breast cancer chemoprevention", "cost-effectiveness of chemoprevention", and "breast cancer costeffectiveness analysis". While selection of papers was not systematic, the literature available in this field is relatively sparse and therefore all cost-effectiveness analyses of chemoprevention drugs for the prevention of breast cancer identified were included. As research over the past 15 years has further clarified the true risk for side effects and the length of time of the protective effect of chemoprevention, we will reassess the findings of these articles in light of new information. The purpose of this review is to analyze the available literature on the costeffectiveness of tamoxifen, raloxifene, and Als for the primary prevention of breast cancer to illustrate how the cost-effectiveness of these pills has changed over time. The review will conclude by suggesting potential areas for future research.

\section{Tamoxifen}

Tamoxifen is a SERM that was initially investigated as a contraceptive in the 1960s. While tamoxifen was a failure as a contraceptive, by the 1970s the drug had become part of the standard of care for the treatment of ER-positive breast cancers. In the 1980s, research on the use of the SERM as a chemopreventive agent began and, in 1998, the Breast Cancer Prevention Trial (BCPT) or the National Surgical Adjuvant Breast and Bowel Project (NSABP-P1) trial showed that tamoxifen use for 5 years could significantly decrease the incidence of breast cancer among highrisk women. ${ }^{6,13}$ Women were considered high risk and therefore eligible for the study if they were older than 60 or between the ages of 35 and 59 with a history of lobular carcinoma in situ, atypical hyperplasia, or a 5-year risk for breast cancer of at least $1.66 \% .{ }^{13}$ Based on this study, the FDA approved tamoxifen for the prevention of breast cancer in high-risk women, although the trial did not at the time confirm that this decreased risk lasted once women ceased tamoxifen use. ${ }^{19}$ Several studies have since corroborated that tamoxifen use for 5 years reduces the risk for breast cancer among high-risk women by $30-40 \% .22-24$ Long-term followup through the International Breast cancer Intervention Study (IBIS-I) trial suggested that risk reduction could last for up to 20 years. ${ }^{8}$ This decreased risk for breast cancer incidence comes with a heightened risk for endometrial cancer and pulmonary embolism, though long-term data indicates that these side effects are relatively rare with an elevated risk only during active treatment. ${ }^{17,24}$ The most frequently seen side effects are menopausal symptoms, such as hot flashes and vaginal symptoms. ${ }^{25,26}$ considering this side-effect profile, the population that tends to benefit the most from tamoxifen are high-risk women between 35 to 50 years of age and women who have had a hysterectomy.17 since 1998, there have been several studies of the cost-effectiveness of tamoxifen for the prevention of breast cancer, both by itself and in comparison to other pills and prevention strategies.

The first few comprehensive analyses of the cost-effectiveness of tamoxifen were published in the early 2000s. These studies were important in determining which women would benefit most from tamoxifen treatment given the perceived risk for negative side effects and the lack of long-term outcome data available at the time from the BCPT. ${ }^{19}$ A study published by Hershman et al. in 2002 found that tamoxifen was particularly cost-effective for high-risk women who started treatment early in life, even if the protective effects only lasted while the pill was being taken. The model was built based on an analysis of women participating in the BCPT, with risk for side effects and survival projections pulled from the Surveillance, Epidemiology, and End Results (SEER) data from 1993 to 1997. Tamoxifen was most cost-effective among 35 and 50-year-old women with a history of atypical hyperplasia, with a survival benefit of 202 days and 89 days and a cost of $\$ 9,777$ and $\$ 26,990$ per QALY, respectively, well below a standard threshold value of $\$ 50,000$. Among 35 and 50-year-old women with a 5-year risk for breast cancer greater than $5 \%$, analysis found a survival benefit of 195 days and 73 days and a cost of $\$ 10,818$ and $\$ 27,901$ per QALY. Based upon sensitivity analysis, their model showed that tamoxifen was cost-effective or even cost-saving (that is, less expensive than placebo) for all high-risk 35 and 50-year-old women if it was assumed that prevention lasted for at least 10 years. The model built by Hershman et al. was fairly sensitive to costs; they found that a decrease in the cost of tamoxifen by $20-40 \%$ would make therapy cost-effective for almost all high-risk groups, including those age 60 or above. ${ }^{18}$ 
In 2003, a model built by Eckermann et al. corroborated much of what was seen in the Hershman study. Their study was also based on the available data from the BCPT and modeled three potential tamoxifen scenarios: using the pill for 5 years with no benefits or risks afterwards, using the pill for 5 years with 10 years of protection, and a model where tamoxifen use only delayed the onset of breast cancer. Costs were specific to Australia. The authors found that tamoxifen therapy was cost-effective if it was assumed that the pills provided risk reduction for 5 or 10 years, at $\$ 38,271$ and $\$ 19,345$ (in Australian dollars) per QALY, respectively. However, in the model where tamoxifen only delayed the onset of breast cancer, the risks for tamoxifen therapy outweighed the benefits with a cost per QALY of $\$ 199,149$. Sensitivity analysis showed that cost-effectiveness improved as 5-year risk for breast cancer increased, however, if 5-year risk was reduced to $1.67 \%$, the minimum to be considered eligible for chemoprevention in the BCPT, the cost per QALY was $\$ 123,000$. The studies by Hershman et al. and Eckermann et al. were similar in that both concluded that the cost-effectiveness of tamoxifen depended upon the long-term outcomes of treatment on patient survival. However, these outcomes were unknown at the time both analyses were conducted, since the data had not matured in the clinical trials. Interestingly, the model showed that most of the benefits of tamoxifen therapy came from the few potentially prevented breast cancers having relatively more severe outcomes than the side effects; that is, the benefits to the few individuals who avoided breast cancer were greater than the harms of side effects to the rest of the modeled population. ${ }^{19}$

Given their date of publication, these earlier studies had to rely on assumptions regarding the long-term outcomes of tamoxifen use and build models to reflect what was believed to be the most likely results of therapy. In 2006, Melnikow et al. revisited the cost-effectiveness of tamoxifen using updated costs and outcomes data from the BCPT. Their model incorporated a greater range of cost information from both Canadian and US healthcare systems, as well as data on the risk for ER-negative breast cancers among women taking tamoxifen. Tamoxifen was compared with placebo and 5-year risk for breast cancer was varied between 1-5\%. In contrast to previous studies, the model built by Melnikow et al. assumed that breast cancer prevention only lasted for the duration of treatment and showed that the tamoxifen was unlikely to be cost-effective for all but the most high-risk women, those with a 5-year breast cancer risk for $5 \%$ or greater. For women with an intact uterus and a $1.67 \% 5$-year risk for breast cancer, the model estimated tamoxifen therapy would lead to a benefit of 1.6 days and a cost per life year of $\$ 1,335,690$. For women with a uterus, the tamoxifen price necessary to achieve $\$ 100,000$ per life year saved, a higher but generally accepted cost threshold, would be $\$ 144$ per year, while the average health maintenance organization (HMO) price in the US was $\$ 415$ at the time. The low benefit to women with a 5-year breast cancer risk for $1.67 \%$ was largely due to the risk for side effects; a 3-4 \% 5-year risk for breast cancer was the minimum necessary for there to be benefits to tamoxifen therapy. ${ }^{27}$ The discrepancy between these findings and previous studies indicated the need to develop a more robust and validated model of the cost-effectiveness of tamoxifen.

The 2006 analysis by Melnikow et al. was followed by a second paper by the same authors in 2008. The authors sought to analyze how the costeffectiveness of tamoxifen would shift if utility values for menopausal symptoms were incorporated into their model. In some types of analysis, these outcomes are considered to have a relatively minor impact on patient health and are not weighted as highly. Melnikow et al. conducted interviews in women with a $1.67 \%$ or higher 5 -year breast cancer risk to determine the utility values of menopausal symptoms and incorporated these values into a model following 50-year-old women with a $1.67 \%$ and $3 \%$-year risk for breast cancer. As with their previous study, the authors found that tamoxifen for chemoprevention was not cost-effective. Among women with a uterus and a 1.67 \% 5-year risk for breast cancer, tamoxifen was shown to have a negative benefit of 9.3 days lost; among women without a uterus, the benefit was only 6.8 days at a cost per QALY of $\$ 300,030$. In the group with a $3 \% 5$-year risk, costs per QALY were $\$ 167,718$ and $\$ 68,262$ for women with and without a uterus, respectively. When a "global utility" rating that weighed participants overall preferences towards tamoxifen, instead of a separate utility for each outcome, was incorporated into the analysis, using the pill resulted in a loss of almost an entire year for all groups. While this study reinforced the previous findings by Melnikow et al., it also indicated the need to use highly validated measurements of patient preferences with cost-effectiveness analysis. ${ }^{28}$

In 2011, Noah-Vanhoucke et al. revisited the cost-effectiveness of tamoxifen. The model they developed used outcomes data pulled from a meta-analysis of the long-term use of tamoxifen for adjuvant therapy. Breast cancer incidence and survival was modeled using the Archimedes Breast Cancer Model, a mathematical model of the outcomes of breast cancer validated against SEER. ${ }^{20}$ Their model averaged the risks for side effects from these studies and incorporated the established minimum 10-year prevention benefit of tamoxifen. Utilities were calculated similarly to Melnikow et al. both with and without adjustment for common menopausal symptoms. Their analysis found that there was a moderate benefit to tamoxifen therapy for women at low to average risk for breast cancer (5-year risk <1.67\%) and that these benefits only increased for higher-risk populations. Tamoxifen therapy was cost-saving for women with a 5-year breast cancer risk greater than $1.67 \%$ and costs remained well below acceptable benchmarks even among populations with a relatively low 5-year Gail risk score of $0.8 \%$. In sensitivity analysis, their model remained robust when costs of both tamoxifen and cancer treatment were increased by up to 3 times. When the risk for all side effects was increased to the upper limit of available estimates, tamoxifen was no longer costeffective at any risk levels, however, therapy remained cost-effective when risk for any one side effect was increased. The ultimate result of the model was that tamoxifen was cost-effective and that benefits outweighed side effects for all postmenopausal women younger than 55 at a $1.67 \%$ 5-year risk for breast cancer. ${ }^{20}$

Several studies have also examined the cost-effectiveness of tamoxifen for chemoprevention in more specific populations, such as those with $B R C A 1 / B R C A 2$ mutations. An analysis by Anderson et al. compared tamoxifen chemoprevention to surgical interventions for the prevention of breast cancer in women with BRCA1/BRCA2 mutations. Generally, it was found that chemoprevention was always more costly with fewer QALYS saved compared with surgical interventions in women with mutations that significantly increased the risk for breast and ovarian cancer. This was largely because prophylactic mastectomy and bilateral salpingo-oophorectomy reduced breast cancer risk by $90 \%$ and $53 \%$, respectively, while the upper estimate for risk reduction from tamoxifen is only $49 \% .^{17,29}$ 
Table 1: Selected Information on the Cost-effectiveness of Tamoxifen and Raloxifene for Chemoprevention

\begin{tabular}{|c|c|c|c|c|c|}
\hline Author, Year & Model Information & Intervention & Population & $\begin{array}{l}\text { 5-Year Breast } \\
\text { Cancer Risk }\end{array}$ & Cost/QALY \\
\hline \multicolumn{6}{|l|}{ Tamoxifen } \\
\hline Hershman, $2002^{18}$ & $\begin{array}{l}\text { Markov model following population similar to BCPT } \\
\text { with outcomes from SEER until age } 100 \text { or death, } \\
\text { assuming protection lasts } 10 \text { years }\end{array}$ & $\begin{array}{l}\text { Tamoxifen for } 5 \text { years } \\
\text { vs placebo }\end{array}$ & $\begin{array}{l}\text { Age } 35-49 \\
50-59 \\
>60\end{array}$ & $\begin{array}{l}>5.0 \% \\
>5.0 \% \\
>5.0 \%\end{array}$ & $\begin{array}{l}\text { Cost-savinga } \\
\$ 11,237 \\
\$ 33,704\end{array}$ \\
\hline Eckermann, $2003^{19}$ & $\begin{array}{l}\text { Markov model following population similar to BCPT } \\
\text { for lifetime, assuming protection lasts } 5 \text { years }\end{array}$ & $\begin{array}{l}\text { Tamoxifen for } 5 \text { years } \\
\text { vs placebo }\end{array}$ & $\begin{array}{l}\text { Age distribution } \\
\text { follows BCPT }\end{array}$ & $\begin{array}{l}1.66 \% \\
3.3 \% \\
6.7 \%\end{array}$ & $\begin{array}{l}\$ 123,000 \\
\$ 38,000 \\
\$ 13,000\end{array}$ \\
\hline Melnikow, $2006^{27}$ & $\begin{array}{l}\text { Markov model following population similar to BCPT } \\
\text { with outcomes from SEER at age } 50-100 \text { or death }\end{array}$ & $\begin{array}{l}\text { Tamoxifen for } 5 \text { years } \\
\text { vs placebo }\end{array}$ & $\begin{array}{l}\text { Age } 50, \text { uterus intact } \\
\text { Age } 50, \text { no uterus }\end{array}$ & $\begin{array}{l}1.67 \% \\
5.0 \% \\
1.67 \% \\
5.0 \%\end{array}$ & $\begin{array}{l}\$ 1,355,690 \\
\$ 64,788 \\
\$ 177,116 \\
\$ 46,954\end{array}$ \\
\hline Anderson, $2006^{29}$ & $\begin{array}{l}\text { Markov model following population similar to BCPT } \\
\text { with outcomes from SEER at age 35-70 or death }\end{array}$ & $\begin{array}{l}\text { Tamoxifen for } 5 \text { years } \\
\text { vs surgical intervention } \\
\text { and surveillance }\end{array}$ & $\begin{array}{l}\text { Age } 35 \text { with } \\
\text { BRCA1/BRCA2 }\end{array}$ & $\begin{array}{l}\text { BRCA1 } \\
\text { BRCA2 }\end{array}$ & $\begin{array}{l}\text { Dominated } \\
\text { Dominated }^{b}\end{array}$ \\
\hline Melnikow, $2008^{28}$ & $\begin{array}{l}\text { Model constructed as in Melnikow (2006), utilities } \\
\text { generated specifically for this study using standard } \\
\text { gamble method }\end{array}$ & $\begin{array}{l}\text { Tamoxifen for } 5 \text { years } \\
\text { vs placebo }\end{array}$ & $\begin{array}{l}\text { Age } 50, \text { uterus intact } \\
\text { Age } 50, \text { no uterus }\end{array}$ & $\begin{array}{l}1.67 \% \\
3.0 \% \\
1.67 \% \\
3.0 \%\end{array}$ & $\begin{array}{l}\text { Dominated } \\
\$ 167,718 \\
\$ 300,030 \\
\$ 68,262\end{array}$ \\
\hline $\begin{array}{l}\text { Noah-Vanhoucke, } \\
2011^{20}\end{array}$ & $\begin{array}{l}\text { Archimedes Breast Cancer Model following population } \\
\text { from NHANES with outcomes from SEER until death }\end{array}$ & $\begin{array}{l}\text { Tamoxifen for } 5 \text { years } \\
\text { vs placebo }\end{array}$ & Age $<50$ & $\begin{array}{l}\geq 0.8 \% \\
\geq 1.66 \% \\
\geq 2.0 \%\end{array}$ & $\begin{array}{l}\$ 12,863.31 \\
\$ 1,693.66 \\
\text { Cost-savinga }\end{array}$ \\
\hline \multicolumn{6}{|l|}{ Raloxifene } \\
\hline Armstrong, $2001^{21}$ & $\begin{array}{l}\text { Markov model validated against data from National Center } \\
\text { for Health Statistics, until age } 101 \text { or death }\end{array}$ & $\begin{array}{l}\text { Raloxifene for } 10 \text { years or } \\
\text { less vs HRT }\end{array}$ & Age 50 & $\begin{array}{l}10 \%^{*} \\
15 \%^{*} \\
30 \%^{*}\end{array}$ & $\begin{array}{l}\text { Dominated } \\
\$ 43,056 \\
\$ 3,830\end{array}$ \\
\hline Ivergård, 201032 & $\begin{array}{l}\text { Markov model following population of Caucasian women } \\
\text { with outcomes from literature until age } 100 \text { or death }\end{array}$ & $\begin{array}{l}\text { Raloxifene for } 5 \text { years } \\
\text { vs placebo }\end{array}$ & $\begin{array}{l}\text { Age } 55,10 \text {-year risk for } \\
\text { fracture } 10-14.9 \%\end{array}$ & $\begin{array}{l}1.0 \% \\
3.0 \% \\
5.0 \%\end{array}$ & $\begin{array}{l}\$ 76,171 \\
\$ 42,561 \\
\$ 27,490\end{array}$ \\
\hline
\end{tabular}

${ }^{\star}$ Lifetime Breast Cancer Risk; alnterventions that are cost-saving are those that cost less than placebo/no treatment; ${ }^{b}$ Interventions that are dominated do not result in increased life years or cost-savings. BCPT = Breast Cancer Prevention Trial; HRT = hormone replacement therapy; NHANES $=$ National Health and Nutrition Examination Survey; QALY = qualityadjusted life years; SEER = Surveillance, Epidemiology, and End Results.

Selected results from these studies are summarized in Table 1. While the most recent study indicates that tamoxifen for chemoprevention is cost-effective, the wide range of cost per QALY ratios, from $\$ 1,600$ to over $\$ 1,300,000$, to treatment being cost-saving or dominated by placebo, indicate the lack of consensus in the available literature. Costeffectiveness models are highly sensitive to the costs, utilities, and outcomes input by researchers as well as the assumptions that must be made to fully capture all aspects of breast cancer chemoprevention. As additional data about the long-term outcomes of tamoxifen for chemoprevention, patient utility towards side effects, and costs of breast cancer care become available, it will be necessary for researchers to build new models to effectively guide policy.

\section{Raloxifene}

Like tamoxifen, raloxifene is a SERM that was initially investigated for purposes other than breast cancer prevention. Raloxifene has been shown to decrease the risk for fractures and increase bone density and in 1997 the FDA approved raloxifene for the treatment of osteoporosis in postmenopausal women. ${ }^{6}$ It was not until 2007 after the completion of the Study of Tamoxifen and Raloxifene (STAR) and Multiple Outcomes of Raloxifene Evaluation (MORE) trials that the FDA approved raloxifene for the primary prevention of breast cancer.6,14,30 Raloxifene has a similar side-effect profile as tamoxifen, including increased risk for thromboembolism and menopausal symptoms. When compared with tamoxifen, raloxifene has only $81 \%$ of the efficacy of tamoxifen for breast cancer risk reduction. ${ }^{11}$ However, raloxifene use is not associated with an increased risk for endometrial cancer or cataracts as with tamoxifen, or an increased risk for bone fractures as seen with Als. These factors make raloxifene an attractive option for chemoprevention, particularly among women who have not had a hysterectomy or who are at high risk for osteoporosis. As with the other options for chemoprevention, uptake of raloxifene among high-risk women has been low. ${ }^{17}$

Several analyses of the cost-effectiveness of raloxifene have been published over the last decade and a half, however, most of these studies have focused on fracture risk reduction with relatively few addressing the use of raloxifene for chemoprevention. Studies that analyze the costeffectiveness of raloxifene for chemoprevention were usually based on models that included risk reduction for other diseases, like osteoporosis or heart disease. A paper by Armstrong et al. in 2001 compared raloxifene and hormone replacement therapy (HRT) for the prevention of breast cancer, osteoporosis, and coronary heart disease. Their model followed healthy 50-year-old women taking raloxifene or HRT for 5 years, 10 years, and lifetime. Both treatments were cost-effective compared with placebo. However, among women at average risk for breast cancer and coronary heart disease, HRT was more cost-effective than raloxifene if taken for 
an entire lifetime assuming, based on available data, that raloxifene did not lead to a sustained reduction in breast cancer rates. In the 5- and 10-year treatment models, raloxifene was more cost-effective than HRT, at a cost per QALY of $\$ 43,056$ and $\$ 3,830$ among women with a lifetime breast cancer risk for $15 \%$ and $30 \%$, respectively. The model was highly sensitive to risk for coronary heart disease and breast cancer risk; if HRT increased the risk for breast cancer or was not as effective at preventing heart disease, raloxifene was usually more cost-effective. The authors noted that their model was limited by the lack of data available on the long-term outcomes of raloxifene for chemoprevention. ${ }^{21}$ This paper predates the results of the Women's Health Initiative (WHI) study on HRT, which indicated that HRT actually increased the risk for coronary heart disease in women compared with placebo. ${ }^{31}$ Incorporating the results of this analysis would potentially show increased cost-effectiveness for raloxifene if it were performed again.

A 2010 paper by Ivergård et al. examined raloxifene for both breast cancer prevention and osteoporosis. Their model followed women at ages 55, 60, and 65 with 5-year breast cancer risk for 1-5\% and 10-year fracture risk for $5-19.9 \%$ and sought to identify which combination of risk values made raloxifene most cost-effective. As the National Osteoporosis Foundation recommends treatment for osteoporosis when 10-year fracture risk is at least $20 \%$, the authors were primarily interested in the outcomes of raloxifene use among women below this risk threshold. ${ }^{32}$ Raloxifene was compared with no treatment in the model, as there was disagreement in the literature over whether alendronate for osteoporosis would be a comparable alternative. While holding fracture risk constant, raloxifene taken for up to 5 years was found to be cost-effective, with significant benefits in QALYs among all groups of women. For example, among 55, 60, and 65-year-old women with a 10-14.9\% 10-year fracture risk and a $3 \% 5$-year breast cancer risk, the cost per QALY was \$42,561, $\$ 43,276$, and $\$ 49,202$, respectively. QALYs gained increased with breast cancer risk, however, increases in QALYs were less steep with age; that is, older women saw benefits from raloxifene use, but at less-steep rates than younger women. While there were still benefits to the women with the lowest rates of fracture and breast cancer risk in sensitivity analysis, the treatment was no longer cost-effective at a cost threshold of $\$ 50,000$. However, raloxifene was cost-effective for women at all examined ages with a 5-year breast cancer risk for at least $4 \%$ regardless of fracture risk rate. Similarly, raloxifene was cost-effective or almost cost-effective for all women at a fracture risk rate of 15-19.9\%, regardless of 5-year breast cancer risk. ${ }^{32}$

Selected results from these two studies are summarized in Table 1. As with the analyses of tamoxifen, the cost-effectiveness of raloxifene was highly sensitive to the costs and outcomes selected by researchers. As raloxifene is frequently prescribed for the prevention of fractures, most cost-effectiveness analyses for this intervention do not focus solely on chemoprevention. While the STAR trial compared the outcomes of tamoxifen and raloxifene, there is a lack of available literature on how these two interventions compare under cost-effectiveness analysis. An analysis of chemoprevention use in Australia found that both treatments were cost-effective among women with a $1.67 \%$ 5-year risk for breast cancer and that raloxifene may be more cost-effective than tamoxifen among high-risk postmenopausal women. ${ }^{33}$ Future models incorporating both tamoxifen and raloxifene for chemoprevention would be useful for understanding which treatments are most costeffective for certain populations.

\section{Aromatase Inhibitors}

There are currently several classes of Als under investigation for the prevention of breast cancer, most notably anastrozole and exemestane. Als work by blocking the synthesis of estrogen by inhibiting the enzyme aromatase and are only effective in preventing ER-positive breast cancers in postmenopausal women. Like tamoxifen, Als are also used for the treatment of ER-positive breast cancer. While none are FDA approved for breast cancer prevention, evidence from recent studies suggests that both may decrease breast cancer incidence.? The MAP-3 trial demonstrated that exemestane decreased breast cancer risk by up to $65 \%$ among highrisk postmenopausal women. ${ }^{9}$ The IBIS-II trial indicated that anastrozole may decrease the risk for breast cancer by up to $53 \%$ in postmenopausal women, aged 40-70 with a 5-year risk for breast cancer higher than the general population. ${ }^{10}$ Als tend to have a different side-effect profile with no increased risk for endometrial cancer or thromboembolic events, although there is evidence of an increased, but not statistically significant, risk for fracture. ${ }^{9}$ One study has indicated that use of exemestane for at least 2 years may lead to loss of bone density. ${ }^{34}$ The populations that stand to benefit most from Als for the prevention of breast cancer are postmenopausal women at high-risk for breast cancer with no history of osteoporosis. ${ }^{7}$ There are currently no available studies comparing the effectiveness of Als to SERMS for the prevention of breast cancer. While there are several studies available on the cost-effectiveness of Als for adjuvant therapy, no literature has been published examining the costeffectiveness of these drugs for chemoprevention.

In 2013, John-Baptiste et al. published a systematic review of the available literature comparing the cost-effectiveness of tamoxifen and Als for adjuvant therapy. ${ }^{35}$ Their analysis found that many of these cost-effectiveness studies showed poor sensitivity and were not widely applicable across different subgroups. Furthermore, many studies extrapolated the health effects of Als or assumed an increased overall survival beyond what has been validated in randomized controlled trials. Thus, while many analyses showed that Als were more cost-effective than tamoxifen for the treatment of breast cancer, providers should be wary in using these studies to guide policy. ${ }^{35}$ While cost-effectiveness analyses can be useful for directing practice, any future studies of the cost-effectiveness of Als for chemoprevention should attempt to avoid the pitfalls that have been identified in the adjuvant studies.

\section{Conclusions}

As healthcare costs increase and the ability to screen women for breast cancer risk improves, it will be critical for providers to be able to compare the cost-effectiveness of different chemoprevention regimens. The available literature on this topic is relatively sparse. Many of the analyses included in this review are from the early 2000s, before longterm outcome data on chemoprevention and generic versions of these medications were available. For tamoxifen, the risk for severe side effects has led to a wide range of cost-effectiveness estimates among women at high-risk for breast cancer. While the most recent analyses indicate that tamoxifen is cost-effective in women with a 5-year breast cancer risk for $1.67 \%$ or higher, it will be necessary to revisit these studies and compare tamoxifen with other chemoprevention options as more data become 
available. Similarly, raloxifene has been found to be a cost-effective option for postmenopausal women at high-risk for breast cancer, but all the available cost-effectiveness papers have tended to focus on the use of the drug for fracture risk reduction. There are no papers available on the cost-effectiveness of Als for breast cancer risk reduction, however, a systematic review has indicated that studies comparing Als to tamoxifen for adjuvant therapy tend to inadequately address uncertainty. As longterm follow-up data from the chemoprevention trials of Als emerge, it will be important to compare these options to both of the SERMs.

Costs of cancer care have continued to grow dramatically over the past several years, and it is estimated that this trend will continue. ${ }^{4}$ The rise in the cost of breast cancer care is driven both by the high expense of therapy, as well as the length over which treatment needs to occur. ${ }^{36}$ Generic versions of tamoxifen, raloxifene, and the Als are available, and a recent paper by Hershman et al. has established that lower costs of copayments for adjuvant hormonal therapy may increase adherence to treatment. ${ }^{37}$ Further research into how decreased costs of chemoprevention medications improve uptake and adherence could potentially have an impact on the overall cost-effectiveness of this preventative therapy.

There is no established association between uptake of chemoprevention and reduction in breast cancer-related deaths, that is, use of tamoxifen or raloxifene for prevention of breast cancer does not reduce fatalities from breast cancer. By preventing breast cancers before they occur, chemoprevention has the potential to reduce costs associated with breast cancer treatment. Establishing whether or not chemoprevention is cost-effective is critical to determining whether preventative treatment for breast cancer results in improved cost and health outcomes for both patients and healthcare systems
1. Centers for Disease Control and Prevention. Cancer among women, 2014. Available at: http://www.cdc.gov/cancer/dcpc/ data/women.htm (accessed August 9, 2015)

2. Centers for Disease Control and Prevention. Breast cancer statistics, 2014. Available at: http://www.cdc.gov/cancer/breast/ statistics/ (accessed August 9, 2015)

3. Barron JJ, Quimbo R, Nikam PT, Amonkar MM, Assessing the economic burden of breast cancer in a US managed care population, Breast Cancer Res Treat, 2008:109:367-77.

4. Mariotto AB, Robin Yabroff $K$, Shao $Y$, et al., Projections of the cost of cancer care in the United States: 2010-2020, J Nat/ Cancer Inst, 2011;103:117-28.

5. Ong M-S, Mandl KD, National expenditure for false-positive mammograms and breast cancer overdiagnoses estimated at $\$ 4$ billion a year, Health Aff, 2015;34:576-83.

6. Maximov PY, Lee TM, Jordan VC, The discovery and development of selective estrogen receptor modulators (SERMS) for clinical practice, Curr Clin Pharmacol, 2013;8:135-55.

7. Stickeler E, Fehm T, Schutz F, Thill M, Aromatase inhibitors in the treatment and prevention of breast cancer, Breast Care, 2015;10:141-2.

8. Cuzick J, Sestak I, Cawthorn S, et al., Tamoxifen for prevention of breast cancer: extended long-term follow-up of the IBIS-I breast cancer prevention trial Lancet Oncol, 2015:16:67-75.

9. Goss PE, Ingle JN, Cheung AM, et al., Exemestane for breastcancer prevention in postmenopausal women, N Engl J Med 2015;364:2381-91.

10. Cuzick J, Sestak I, Forbes JF, et al., Anastrozole for prevention of breast cancer in high-risk postmenopausal women (IBIS-II): An international, double-blind, randomised placebo-controlled trial, Lancet, 2014:383:1041-8.

11. Wickerham DL, Cecchini RS, Vogel VG et al., Final updated results of the NRG Oncology / NSABP Protocol P-2 : Study of tamoxifen and raloxifene ( STAR ) in preventing breast cancer. Presented at ASCO Annual Meeting, Chicago, 2015.

12. Ropka ME, Keim J, Philbrick JT, Patient decisions about breas cancer chemoprevention: A systematic review and metaanalysis, J Clin Oncol, 2010;28:3090-5.

13. Fisher B, Costantino JP, Wickerham DL, et al., Tamoxifen for the prevention of breast cancer: Current status of the Nationa Surgical Adjuvant Breast and Bowel Project P-1 study, J Natl
Cancer Inst, 2005;97:1652-62.

14. Vogel VG, Costantino JP, Wickerham DL, et al., Update of the National Surgical Adjuvant Breast and Bowel Project Study of Tamoxifen and Raloxifene (STAR) P-2 Trial: preventing breast cancer, Cancer Prev Res, 2010;3:696-706.

15. Freedman AN, Graubard BI, Rao SR, et al., Estimates of the number of US women who could benefit from tamoxifen for breast cancer chemoprevention, J Natl Cancer Inst, 2003:95:526-32

16. Ravdin PM, The lack, need, and opportunities for decisionmaking and informational tools to educate primary-care physicians and women about breast cancer chemoprevention, Cancer Prev Res (Phila), 2010;3:686-8.

17. Crew KD, Addressing barriers to uptake of breast cancer chemoprevention for patients and providers, $A s c o E d u c B$ 2015:50-8.

18. Hershman D, Sundararajan $\mathrm{V}$, Jacobson IS, et al., Outcomes of tamoxifen chemoprevention for breast cancer in very high-risk women: A cost-effectiveness analysis, J Clin Oncol, 2002;20:9-16.

19. Eckermann SD, Martin a J, Stockler MR, Simes RJ, The benefits and costs of tamoxifen for breast cancer prevention Aust N Z Public Health, 2003;27:34-40.

20. Noah-Vanhoucke J, Green LE, Dinh TA, et al., Cost-effectiveness of chemoprevention of breast cancer using tamoxifen in a postmenopausal US population, Cancer, 2011:117:3322-31.

21. Armstrong $K$, Chen TM, Albert D, et al., Cost-effectiveness of raloxifene and hormone replacement therapy in postmenopausal women: impact of breast cancer risk, Obstet Gynecol, 2001;98:996-1003

22. Powles TJ, Ashley S, Tidy A, et al., Twenty-year follow-up of the Royal Marsden randomized, double-blinded tamoxifen breast cancer prevention trial, J Nat/ Cancer Inst, 2007:99:283-90.

23. Veronesi U, Maisonneuve P, Rotmensz N, et al., Tamoxifen for the prevention of breast cancer: Late results of the Italian randomized tamoxifen prevention trial among women with hysterectomy, J Natl Cancer Inst, 2007;99:727-37.

24. Cuzick J, Forbes JF, Sestak I, et al., Long-term results of tamoxifen prophylaxis for breast cancer-96-month follow-up of the randomized IBIS-I trial, J Nat/ Cancer Inst, 2007:99:272-82.

25. Cuzick J, Sestak I, Bonanni B, et al., Selective oestrogen receptor modulators in prevention of breast cancer: An updated metaanalysis of individual participant data, Lancet, 2013;381:1827-34 26. Donnelly LS, Evans DG, Wiseman J, et al. Uptake of tamoxifen in consecutive premenopausal women under surveillance in a high-risk breast cancer clinic, Br J Cancer, 2014;110:1681-7.

27. Melnikow J, Kuenneth $\mathrm{C}$, Helms $\sqcup$, et al., Chemoprevention: Drug pricing and mortality - The case of tamoxifen, Cancer 2006:107:950-8.

28. Melnikow J, Birch $\mathrm{S}$, Slee $\mathrm{C}$, et al., Tamoxifen for breast cancer risk reduction, Med Care, 2008;46:946-53.

29. Anderson K, Jacobson JS, Heitjan DF, et al., cost-effectiveness of preventative strategies for women with a BRCA1 or a BRCA2 mutation, Ann Intern Med, 2006;144:397-406.

30. Lee WL, Chao HT, Cheng MH, Wang PH, Rationale for using raloxifene to prevent both osteoporosis and breast cancer in postmenopausal women, Maturitas, 2008;60:92-107.

31. Manson J, Hsia J, Johnson KC, et al., Estrogen plus progestin and the risk of coronary heart disease, N Eng J Med, 2003;349:523-34.

32. Ivergård M, Ström O, Borgström F, et al., Identifying costeffective treatment with raloxifene in postmenopausal women using risk algorithms for fractures and invasive breast cancer, Bone, 2010;47:966-74.

33. Taylor PS, Moore PT, Bishop JF, et al., The health economics of chemoprevention for breast cancer in Australia. Available at: www.cancerinstitute.org.au/media/78100/im-2008-01 at: wwW.cancerinstitute.org.au/media/78100/im-2008-01_

34. Cheung AM, Tile L, Cardew S, et al., Bone density and structure in healthy postmenopausal women treated with exemestane for the primary prevention of breast cancer: a nested substudy of the MAP.3 randomised controlled trial, Lancet Oncol, 2012;13:275-84.

35. John-Baptiste AA, Wu W, Rochon P, et al., A systematic review and methodological evaluation of published cost-effectiveness analyses of aromatase inhibitors versus tamoxifen in early stage breast cancer, PLOS One, 2013;8:e62614.

36. Irwin ML, Fabian C, McTiernan A, Improving outcomes for breast cancer survivors, Adv Exp Med Biol, 2015;862:193-212.

37. Hershman DL, Tsui J, Meyer J, et al., The change from brandname to generic aromatase inhibitors and hormone therapy adherence for early-stage breast cancer, I Natl Cancer Inst, 2014:106:dju319. 